\title{
A Mouse Brain Homolog of the Drosophila Shab K+ Channel with Conserved Delayed-Rectifier Properties
}

\author{
Michael D. Pak, ${ }^{1}$ Manuel Covarrubias, ${ }^{1}$ Ann Ratcliffe, ${ }^{1}$ and Lawrence Salkoff ${ }^{1.2}$ \\ 'Department of Anatomy and Neurobiology and 'Department of Genetics, Washington University School of Medicine, St. \\ Louis, Missouri 63110
}

\begin{abstract}
We have cloned and expressed a mouse brain $\mathbf{K}^{+}$channel that is the homolog of the Drosophila Shab $\mathrm{K}^{+}$channel. Mouse and Drosophila Shab $\mathrm{K}^{+}$channels (mShab and IShab, respectively) represent an instance of $K^{+}$channels in distantly related species that are both functionally and structurally conserved; most kinetic, voltage-sensitive, and pharmacological properties are similar for the 2 channels. The greatest functional difference between the currents is recovery from inactivation, which is several times slower for mShab than for $\mathbf{f S h a b}$ currents. In addition to conserved structure, the $m S h a b$ polypeptide has an unusually long nonconserved region at the carboxyl end of the protein. Truncation of 293 residues from the carboxyl end produced no noticeable change in voltage-sensitive, kinetic, or pharmacological properties. Thus, the measured functional properties of mShab are determined by the remaining 564 residues, most of which are conserved. The $m S h a b$ and $f S h a b$ channels are naturally occurring structural variants having substitutions in conserved portions that appear relatively neutral with respect to all measured properties except for, possibly, the rate of recovery from inactivation. The $m S h a b$ current closely resembles a native delayed-rectifier-type potassium current, $I_{k}$, in hippocampal neurons.
\end{abstract}

Potassium channel diversity is generated by an extended gene family encoding homologous channel proteins. An extended gene family consisting of 4 distinct members was originally isolated from Drosophila (Tempel et al., 1987; Kamb et al., 1988; Pongs et al., 1988; Butler et al., 1989; Wei et al., 1990). Each Drosophila gene, Shaker, Shab, Shaw, and Shal, is conserved in mammals (Baumann et al., 1988; Tempel et al., 1988; Frech et al., 1989; Yokoyama et al., 1989; Wei et al., 1990), where some are present as multigene subfamilies.

cDNAs from at least 6 mammalian Shaker subfamily genes have been isolated and expressed in the Xenopus expression system (Baumann et al., 1988; Stuhmer et al., 1988, 1989; Tcmpel et al., 1988; Christie et al., 1989, 1990; McKinnon, 1989;

\footnotetext{
Received May 14, 1990; revised Aug. 30, 1990; accepted Oct. 11, 1990.

We thank A. Butler for technical help and A. Wei, K. Baker, and E. McCleskey for many helpful suggestions. We also thank $J$. Merlie for the generous gift of a cDNA library. This research is supported by NIH 1 RO1NS24785-01 and by grants from the Muscular Dystrophy Association of America and from MonsantoSearle. M.D.P. is supported by Training Grant NS07071-11.

Correspondence should be addressed to Lawrence Salkoff, Department of Anatomy and Neurobiology, Box 8108 -WUMS, 660 South Euclid Avenue, St. Louis, MO 63110 .
}

Copyright $(\mathcal{C} 1991$ Society for Neuroscience $0270-6474 / 91 / 110869-12 \$ 03.00 / 0$
Yokoyama et al., 1989; Chandy et al., 1990; Grupe et al., 1990; Koren et al., 1990; Swanson et al., 1990). However, none of the currents expressed by the mammalian Shaker homologs closely resembles the Shaker current from Drosophila; most mammalian currents of Shaker homologs undergo slow inactivation and have been categorized as "delayed-rectifier" channels. The Drosophila Shaker current, in contrast, is a transient, A-type $\mathrm{K}^{+}$current (Connor and Stevens, 1971; Salkoff, 1983; Solc et al., 1987; Iverson et al., 1988; Timpe et al., 1988a,b). Although one of the mammalian Shaker currents, $R C K 4$, does show rapid inactivation, it differs significantly from the Drosophila A-current in its voltage-sensitive properties (Stuhmer et al., 1989).

We show, however, that the mouse and Drosophila homologs of Shab $\mathrm{K}^{+}$channels ( $m S h a b$ and $f$ Shab) represent an instance of homologs in distantly related species that are functionally as well as structurally conserved. This is true with regard to their voltage-sensitive properties and most kinetic and pharmacological properties. The properties of the $m S h a b$ current also closely match those of one type of delayed rectifier current, $I_{\mathrm{K}}$, observed in the rat hippocampus. Unlike the mammalian Shakcr type of delayed-rectifier currents, which activate very rapidly, $m S h a b$ and $f S h a b$ currents activate more slowly. $m S h a b$ and $f$ Shab currents also differ from the mammalian Shaker currents in that they are insensitive to 4-aminopyridine (4-AP). mShab and $f$ Sh $a b$ currents are distinct from a previously expressed homolog of Drosophila Shab isolated from the rat brain, $d r k 1$ (Frech et al., 1989).

\section{Materials and Methods}

Screening of the mouse brain cDNA library. A mouse brain cDNA library kindly provided by J. Merlie was screened using a degenerate synthetic oligonucleotide probe $\left(\mathrm{CG}^{\mathrm{A}} /{ }_{G} \mathrm{TC}^{\mathrm{A}} /{ }_{G} \mathrm{AA}^{\mathrm{A}} /{ }_{G} \mathrm{AA}^{\mathrm{A}} /{ }_{G} \mathrm{TA}^{\mathrm{T}} /{ }_{C} \mathrm{TC}^{\mathrm{G}} /{ }_{\mathrm{A}} \mathrm{TT}\right)$ based on a highly conserved region ( $m S h a b 1$ residues $81-87$; Fig. 1 ). Oligonucleotides were end labeled with ${ }^{32} \mathrm{P}$ using T-4 nucleotide kinase (Maniatis et al., 1982). Hybridization conditions were $5 \times \operatorname{SSPE}(1.1 \mathrm{M} \mathrm{NaCl}, 60$ mM $\mathrm{NaH}_{2} \mathrm{PO}_{4}, 6 \mathrm{~mm} \mathrm{Na}{ }_{2}$ EDTA, pH 7.7), $5 \times$ Denhardt's, and $0.5 \%$ SDS at $42^{\circ} \mathrm{C}$ for $12 \mathrm{hr}$; filters were washed in $1 \times$ SSC (Maniatis et al., 1982) with $0.1 \%$ SDS at $25^{\circ} \mathrm{C}$. The first $m S h a b$ cDNA isolated was incomplete and was used as a probe, under conditions of high stringency, to isolate the full-length clone $m S h a b 1$. The high-stringency conditions were $5 \times$ SSPE, $5 \times$ Denhardt's, and $0.5 \%$ SDS at $65^{\circ} \mathrm{C}$ for $12 \mathrm{hr}$ followed by a wash in $0.1 \times \mathrm{SSC}$ with $0.1 \%$ SDS at $55^{\circ} \mathrm{C}$.

Nucleotide sequence determination and analysis. The $m S h a b l \mathrm{cDNA}$ was subcloned into both $\mathrm{M} 13 \mathrm{mp} 18$ and pBluescriptII $\mathrm{SK}^{+}$vectors (Stratagene). Both single-stranded and double-stranded DNAs were used as templates, and bidirectional sequencing was performed using the dideoxy technique (Sanger et al., 1977). Sequences were analyzed using MICROGENIE software (Beckman). The truncated $m S h a b 1^{\text {sso. }}$ was sequenced and analyzed in a similar manner.

Vector construction and $c R N A$ synthesis. mShabl, mShabl ${ }^{\text {s.s.5, and }}$ $f$ Shab cDNAs were subcloned into plasmid vectors (pBluescript II SK ${ }^{+}$, 
$-89$

$-60$

* $\pitchfork$

$-30$

$-1$

GAGCTCCCCGCCCCCGCCCGCGGCAGCGGGCCTTGCCGTCGAGTGACAGCGGCCTGGGGGGGCAGGGGGGCGGGGGCGGCCAGACCAGCG

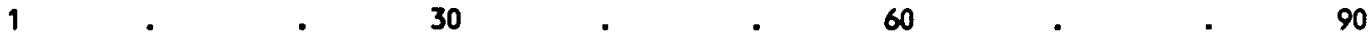
ATGCCGGCGgGCATGACGMGCATGGCTCGCGCTCCACCAGCTCGCTGCCGCCCGAGCCCATGGAGATCGTGCGCAGCAAGGCGTGCTCG

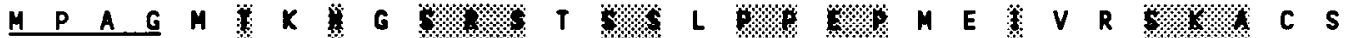

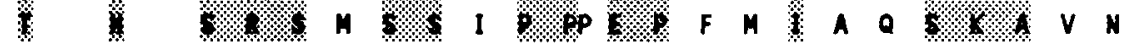

mShab 30

CGCCGGGTGCGCCTCAACGTCGGGGCCTGGCGCACGAGGTGCTGTGGCGCACTCTGGACCGCCTGCCTCGCACGCGGCTGGGCAAGCTC

fShab 276

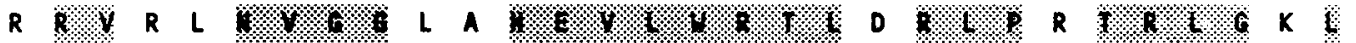

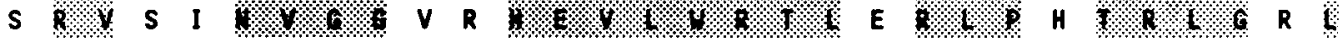

mShab 60

CGgGACTGCAACACGCACGACTCTCTGCTCCAgGTGTGCGACGACTACAGCCTCGAGGACAACGAGTACTTCTTCGACCGCCACCCTGGC

fShab 306

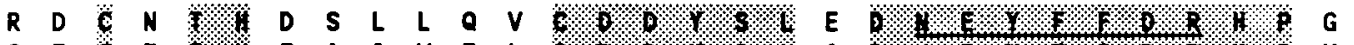

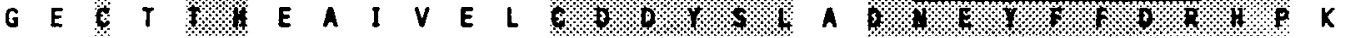

mShab 90

fShab 336

300

330

360

GCCTTCACCTCTATTCTCA

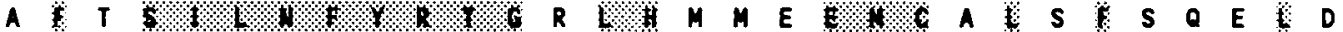

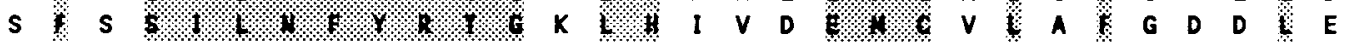

mShab 120

fShab 366

390

420

450

TACTGGGGCATCGATGAGATCTACCTGGAGTCCTGCTGCCAGGCCCGCTACCACCAAAGGAGGAGCAGATGAACGAGGAGCFGAAGCGG

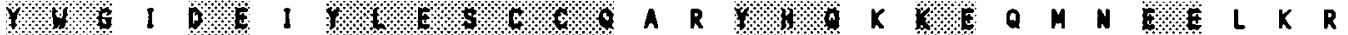
Y106 $V$ D

mShab 150

fShab 396

480

510

540

GAGGCTGAGACGCTGCGGGAGCGGGAGGGCGAGGAGTTCGACAACACGTGCTGTGCTGAGAAGAGGAAGAAACTTTGGGACCTGCTGGAG W 1 E T W $\begin{array}{lllllllllllllllllllllll}0 & S\end{array}$

mShab 180

fShab 426

$570 \quad . \quad 600 \quad 630$ AAGCCCAACTCATCGGTGGCCGCCAAGATCCTGGCCATCATCTCCATCATGTTCATTGTCCTCTCCACCATTGCCCTGTCACTCAACACA

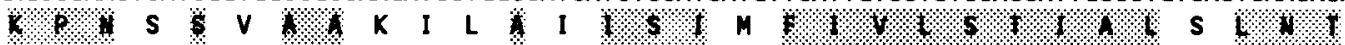

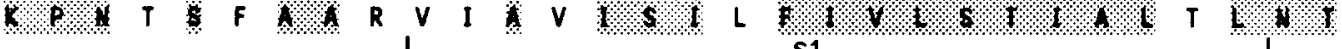

mShab 210

fShab 456

660

690

720

CTGCCTGAGCTACAGAGCCTGGACGAATTCGgCCAGAGCACGgACAACCCGCAGCTGGCACACGTGGAGGCTGTGTGCATCGCGTGGTTC

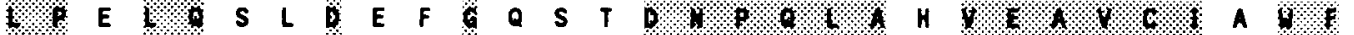

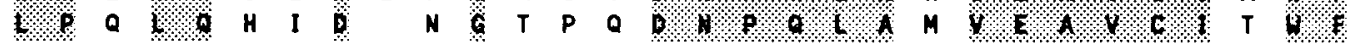
s2

mShab 240

fShab 485

750

780

810

ACCATGgAGTACTTGCTGAGGTTCCTGTCCTCGCCCAAGAAATgGAAGTICTITAAGGGCCCCCTCAACGCCATTGACTTACTGGCCATC

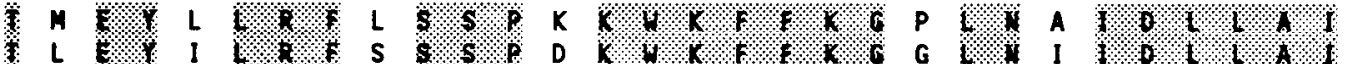

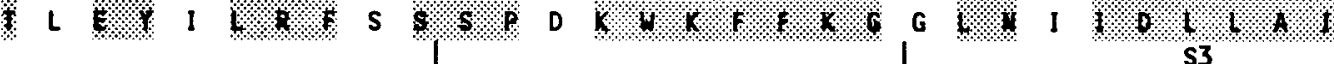

mShab 270

fShab 515

$840 \quad . \quad 870 \quad . \quad 900$ CTGCCCTACTACGTCACCATCTTCCTCACAGAATCCAACAAGAGCGTGCTGCAGTTCCAGAATGTGCGCCGTGTGGTCCAGATCTTCCGC \%

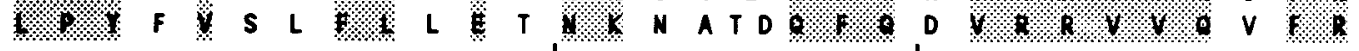

mShab 300

fShab 546

930

960

990

ATCATGCGCATCCTGCGCATCCTGAaGT TGGCCCGCCACTCCACCGGTCTGCAGTCCTTGGGCTTCACGCTGCGCAGGAGCTACAACGAG \%. W. W I

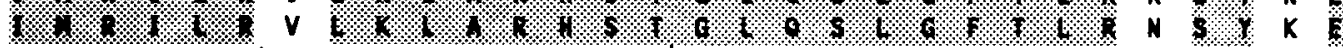

mShab 330 S4

CTGGCCTTGC̈TCATCCTCTICCTCGCCATGGGCATCATGATCTTCTCCAGCCTGGTCTTCTTTGCCGAGAAGGATGAGGATGACACCAAG

CTGGCCTTGCTTCATCCTCTTCCTCGCCATGGGCATCATGATCTTCTCCAGCCTGGTCTTCTTTGCCGAGAAGGATGAGGATGACACCAAG

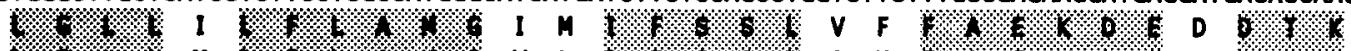

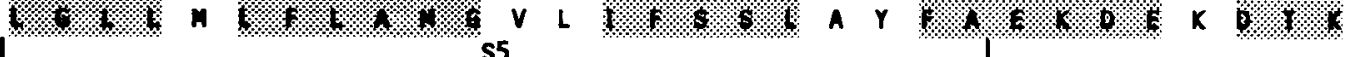

mShab 360

fShab 606

Figure 1. Sequence comparisons of Shab proteins: nucleotide and deduced amino acid sequence of $m S h a b l$ and its comparison to $f$ Shab and $d r k 1$. Identical amino acid residues common to both $m S h a b l$ and $f S h a b$ are shaded. Amino acid residues that differ between $m S h a b l$ and $d r k l$ are shown below the corresponding $m S h a b l$ residues; the $d r k 1$ residues are shown in parentheses. The first stop codon upstream and in frame with the designated translation initiator methionine codon is marked with asterisks $\left.{ }^{* * *}\right)$. The $m$ Shabl amino acid residues $1-4$ (underlined) are not present in the deduced $d r k 1$ protein sequence. The underlined amino acid residues $81-87$ are conserved in most $\mathrm{K}^{+}$channels. The SphI restriction 

S6

fShab 636

1290

1320

1350

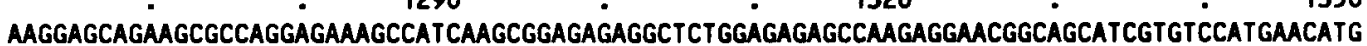

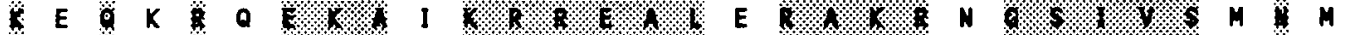
A N O M R R E

mShab 450

tShab 699

1380

1410

1440

AAGGATGCCTTCGCCCGGAGCATCGAGATGATGGACATCGTGGTGGAGAAAMATGGAGAGGGCGTGGCTAMGAAGGACAMGTGCAGGAT

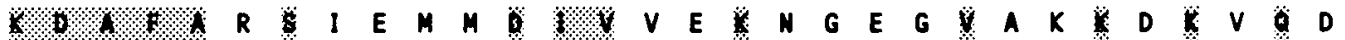
10101\% $K$ S (S)(I)

AACCACCTGTICTCCCAACAAGTGGAAATGGACCAAGAGGGCGCTCTCCGAGACCAGCTCGAGCAAGTCCTITTGAMCCAMGGAGCAGGGA

mShab 420

fShab 666

N H L L S

mShab 480

tShab 730

(drk1)

1560

1590

1620

TCTCCTGAGAAGGCAGGTCCTCGTCTAGTCCGCAGCACITGAACGTCCAGCAGCTGCAAGACATGTACAGCAAGATGGCAAGACCCAG $\begin{array}{llllllllllllllllllllllllllllllll}S & P & E & K & A & R & S & S & S & S & P & Q & H & L & N & V & Q & Q & L & Q & D & M & Y & S & K & M & A & K & T & 0\end{array}$

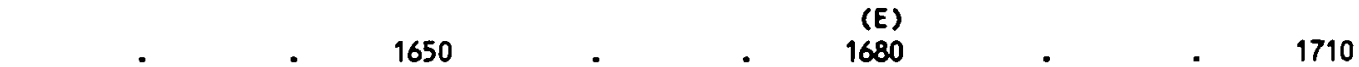
TCTCAACCCATCCTCAACACCAAGGAGATGGCGCCGCAGAGCCAGCCGCAGGAAGAACTGGAGATGGGCAGCATGCCCAGCCCCGTGGCC $\begin{array}{lllllllllllllllllllllllllllllll}S & Q & P & I & L & N & T & K & E & M & A & P & Q & S & Q & P & Q & E & E & L & E & M & G & S & M & P & S & P & V & A\end{array}$ (K) (P)

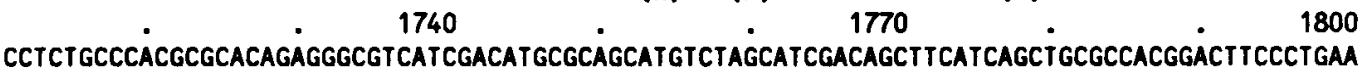
$\begin{array}{llllllllllllllllllllllllllllll}P & L & P & T & R & T & E & G & V & I & D & M & R & S & M & S & S & I & D & S & F & I & S & C & A & T & D & F & P & E\end{array}$ (A)

$1830 \quad$. $\quad 1860 \quad 0 \quad 1890$ GCCACCAGATTCTCCCACAGTCCTCTGGCATCCCTCTCCGGCAAGTCTGGGGGCAGCACAGCCCCGGAGGTGGGCTGGCGGGGGGTICTG A T R F S H S P $L A$ A $S$ L S G K S G G S T A P E V G W R G A L (S) (A) (S)

1920 1950

1980 GGTGCCAGCGGCGGGAGACTCATGGAGACCAACCCCATCCCCGAGGCCAGCCGCTCTGGTTTCTTCGTGGAGAGCCCCCGGAGTTCCATG G A S G G $R$ R L M E (T) (T) AAGACCCACAACCCCATGAAGCTGCGAGCGCTCAAGGTTAACTTCCTGGAGGGCGATCCCACCCCGCTGCTACCGGCTCTGGGCTTGTAT K $T$ T

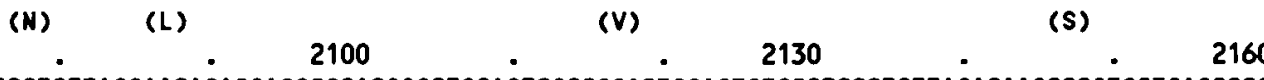
CACGATCCTCTTAGgAaCAGAGGAGGCGCACGgGCTGCAGTCGCCGgACTGGAGTGTGCCTCCCTCTTAGACAAGCCCGTGCTGAGCCCG H D P L R N R G G A R A A V A G L E C A S L L (A)

$21902 \quad 2220 \quad 20250$ GAGTCCTCCATCTACACCACAGCAAGTGCCAGGACGCCCCCTCGCTCCCCAGAGAAACACACAGCAATAGCGTTCAACTTCGAGGCGGG E $\begin{array}{lllllllllllllllllllllllllllll} & S & I & Y & T & T & A & S & A & R & T & P & P & R & S & P & E & K & H & T & A & I & A & F & N & F & E & A & G\end{array}$

mShab 540 (drk1)

mShab 570 (drk1)

mShab 600 (drk1)

mShab 630 (drk1)

mShab 660 (drk1)

mShab 690 (drk1)

mShab 720 (drk1)

mShab 750

2280 2310 2340

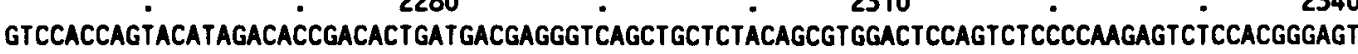
$\begin{array}{llllllllllllllllllllllllllllll}V & H & O & Y & I & D & T & D & T & D & D & E & G & 0 & L & L & Y & S & V & D & S & S & L & P & K & S & L & H & G & S\end{array}$ (H) (P)

mShab 780 (drK1)

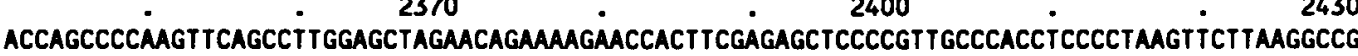

$\begin{array}{llllllllllllllllllllllllllllll}T & S & P & K & F & S & L & G & A & R & T & E & K & N & H & F & E & S & S & P & L & P & T & S & P & K & F & L & R & P\end{array}$ (T)

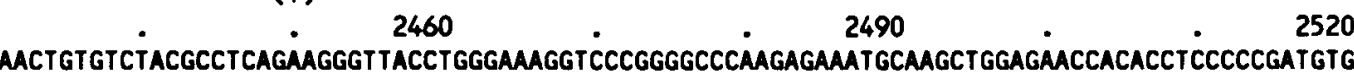

$\begin{array}{llllllllllllllllllllllllllllll} & C & V & Y & A & S & E & G & L & P & G & K & G & P & G & A & 0 & E & K & C & K & L & E & N & H & T & S & P & D & V\end{array}$ (S) (T)

(P)

mShab 840

Cacatgctgcctggggaggagcacacggagcactcggatcagagtaíctga

H M L P G G G A H G S T R D O S I U

mShab 810

(drk1)

(drk1) 


\section{Hydrophilicity Profile}
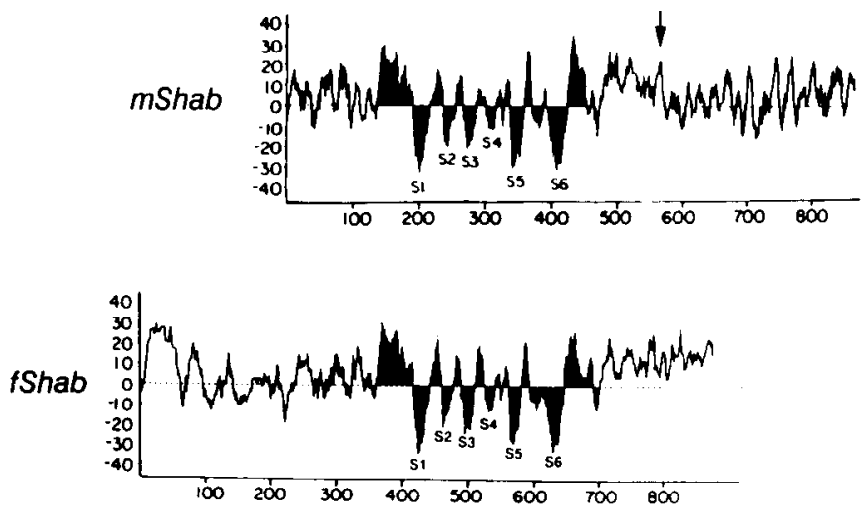

Figure 2. Hydrophilicity profile of $m S h a b l$ and $f$ Shab. Profiles were computed according to Kyte and Doolittle (1982) with a window size of 9 amino acids. Negative (downward) index values indicate hydrophobic groups, while positive values indicate hydrophilic groups. The truncation site to create $m S h a b 1^{\Delta 565}$ is indicated by an arrow.

Stratagene), which served as template for in vitro transcription reactions. The $m S h a b l$ construct included the sequence shown in Figure 1 and an additional 800 base pairs (bp) of a 3' untranslated region. The $f S h a b$ cDNA is the Shabll cDNA (Butler et al., 1990). Expression was enhanced by the replacement of the original translational start site with the consensus translation initiation sequence CCACCATGG (Kozak, 1986). The $m S h a b 1^{\Delta 565}$ cDNA was made from the $m S h a b l$ cDNA by cleaving at the SphI site (Fig. 1, 1691) and removing the downstream sequence. A 200 -bp fragment containing the $3^{\prime}$ untranslated sequence and the translation stop signal was added at the SphI site. The polymerase chain reaction technique (Perkin Elmer; Saiki et al., 1985) was used to synthesize this fragment.

Capped cRNA for both $\mathrm{mShabl}$ and $f$ Shab was synthesized in vitro as previously reported (Wei et al., 1990). The transcription reaction contained $3 \mu \mathrm{g}$ of linearized template DNA, $1 \mathrm{~mm}$ nucleotide triphosphates, $1 \mathrm{~mm} 7 \mathrm{mG}\left(5^{\prime}\right) \mathrm{ppp}\left(5^{\prime}\right) \mathrm{G}$ (cap analog; New England Biolab), and 20 U T3 RNA polymerase, in supplied (Stratagene) transcription buffer and was incubated at $37^{\circ} \mathrm{C}$ for $1 \mathrm{hr}$.

Expression of CRNAs in Xenopus oocytes. Oocytes were injected with $\sim 50 \mathrm{nl}$ (approximately $100 \mathrm{ng}$ ) cRNA in water and incubated in ND96 (as below, plus $1.8 \mathrm{~mm} \mathrm{CaCl}_{2}$ ), supplemented with $2.5 \mathrm{~mm}$ sodium pyruvate, penicillin $(100 \mathrm{U} / \mathrm{ml})$, and streptomycin $(100 \mu \mathrm{g} / \mathrm{ml})$, at $19^{\circ} \mathrm{C}$ for 2-4 d. Prior to injection with cRNA, Xenopus laevis oocytes (stage 4-6) were incubated for $2 \mathrm{hr}$ in collagenase ( $1 \mathrm{mg} / \mathrm{ml}$; type IA, Sigma) in $\mathrm{ND} 96$ without $\mathrm{Ca}^{2+}\left(96 \mathrm{~mm} \mathrm{NaCl}, 2 \mathrm{~mm} \mathrm{KCl}, 1 \mathrm{~mm} \mathrm{MgCl}_{2}, 5 \mathrm{~mm}\right.$ HEPES, pH 7.5). Macroscopic currents were recorded in ND96 using a conventional 2 -microelectrode voltage clamp. One mM 4,4'-diiosothiocyanatostilbene-2,2'-disulfonic acid (DIDS; Calbiochem) was added to block the endogenous $\mathrm{Ca}^{2+}$-dependent $\mathrm{Cl}^{-}$current. Current records were filtered at 0.5 or $1 \mathrm{kHz}$ with an 8 -pole Bessel filter, acquired digitally with CCURRENT, and analyzed using CQUANT (software written by Keith Baker). Curve fitting was done using nonlinear least-square fitting routines (CQUANT and NFIT, Island Products). Recordings were performed at room temperature $\left(21-24^{\circ} \mathrm{C}\right.$ ) or at $15^{\circ} \mathrm{C}$ using a Peltier device (Cambion).

\section{Results}

Isolation and sequencing of Shab clones

A full-length $m$ Shab clone ( $m S h a b l$ ) was isolated by taking advantage of a sequence of 7 amino acids upstream of the S1 transmembrane region found to be conserved in most potassium channels (Fig. 1, mShab 81-87). A degenerate oligonucleotide probe based on these 7 amino acids was used to screen a mouse brain cDNA library. A 1.7-kilobase (kb) 5'-biased cDNA clone was isolated and subcloned into an M13mp18 vector. Singlestranded sequencing using the same degenerate oligonucleotide as a primer revealed homology with the Drosophila Shab gene (Butler et al., 1989). Further sequencing revealed that this clone was truncated at the $3^{\prime}$ end between the $\mathrm{S} 1$ and $\mathrm{S} 2$ transmembrane region by a natural EcoRI site (Fig. 1, 655). A larger cDNA of approximately $3.5 \mathrm{~kb}$ containing the complete translated region was isolated using the $5^{\prime}$-biased cDNA as a probe to screen the same library. The cDNA inserts were subcloned into $\mathrm{M} 13 \mathrm{mp} 18$ and Bluescript vectors for sequencing. The isolation of the Drosophila Shabl1 (fShab) cDNA was reported previously (Butler et al., 1989).

\section{Sequence analysis and primary structure of $\mathrm{mShabl}$}

The full-length $m S h a b$ cDNA, mShabl, has an open reading frame of 2571 nucleotides, which encodes a protein of $857 \mathrm{ami}-$ no acid residues of MW 96,000 (Fig. 1). The putative translation initiation codon is the first ATG triplet upstream in the longest open reading frame. Upstream from this ATG translation start site, the reading frame is closed by a TGA stop signal (Fig. 1). The $5^{\prime}$ untranslated region extends approximately $1.0 \mathrm{~kb}$ upstream of the assigned initiation codon and may not represent the complete $5^{\prime}$ end of the transcript. The sequence surrounding the assigned initiation methionine (CCAGCGATGC) agrees at 6 of 10 sites with the consensus sequence for eukaryotic initiation (GCCA/ ${ }_{\mathrm{G}} \mathrm{CCATGG}$; Kozak, 1986, 1987, 1989); the important purine residue $(G)$ is present at -3 . The open reading frame is terminated by a TGA codon at position 2572 , followed by approximately 800 bp of 3 ' untranslated region. A polyadenylation signal, AATAAA (Proudfoot and Brownlee, 1976) is present, as well as a polyA tail of 22 nucleotides.

The $m S h a b 1$ and $S$ Shab conceptual proteins have very similar hydrophilicity profiles in the central core region containing the 6 putative hydrophobic transmembrane regions S1-S6 (Fig. 2). The similarity of profiles for the intramembrane regions sharply contrasts with the lack of similarity seen in the terminal regions. It can be seen from Figure 2 that the relative position of the conserved core within the protein as a whole differs considerably between $m S h a b l$ and $f S h a b$; for $m S h a b l$, the conserved core is much closer to the $\mathrm{N}$-tcrminal and rescmbles most other cloned $\mathrm{K}^{+}$channels in this regard (Schwarz et al., 1988; Butler et al., 1989; Stuhmer et al., 1989).

The degree of identity between $m S h a b l$ and $f S h a b$ is approximately $70 \%$ over the conserved region (Fig. 1). This is in contrast to an average of approximately $38 \%$ when comparing the percent identity of either Shab protein with a Shaker protein from either fly or mouse (Wei et al., 1990). The total conservation between $m S h a b 1$ and $f S h a b$ over the area compared in Figure 1 is approximately $83 \%$.

Differences are present between $m S h a b l$ and $f$ Shab outside of the central core region. The $m S h a b l$ peptide has an extended C-terminal portion, whereas the $f S h a b$ peptide has a longer $\mathrm{N}$-terminal portion. The homology between $m S h a b l$ and $S h a b$ begins close to the initiator methionine of $m S h a b l$ (Fig. 1; $m S h a b$ 6). Because of the large nonconserved N-terminal extension of fShab (Butler et al., 1989), the conserved portion of the protcin in $f S h a b$ begins 253 residues downstream from the initiator methionine in the fly protein. Notably, the $m S h a b l$ initiation site is at the border of conservation shared by all $4 \mathrm{~K}^{+}$channel subfamilies, Shab, Shaker, Shaw, and Shal.

Asparagine-linked glycosylation consensus sequences $\left(\mathrm{NX}^{\mathrm{T}} / \mathrm{s}\right.$; Kornfeld and Kornfeld, 1985) are found in a similar position 


\section{Activation Properties}

A
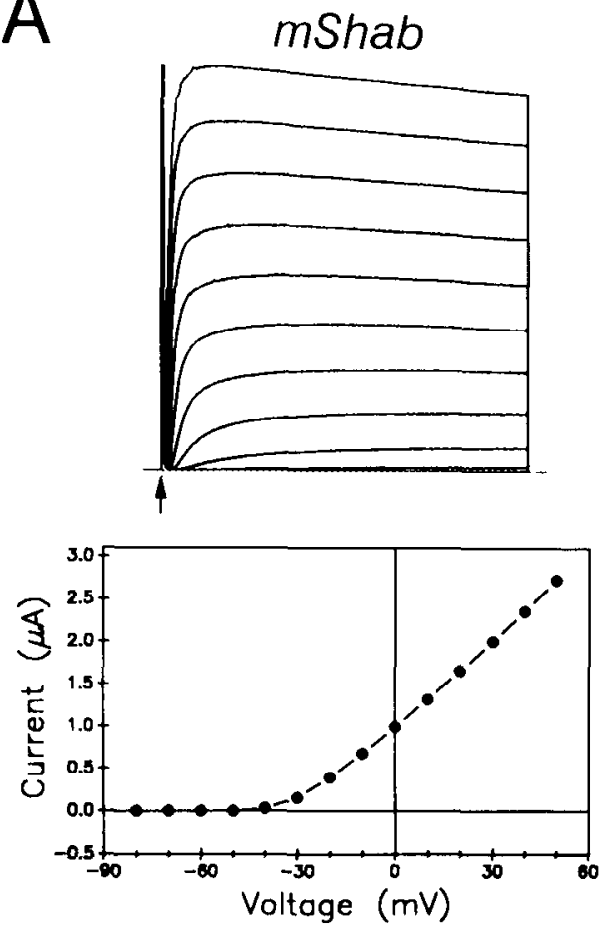

B

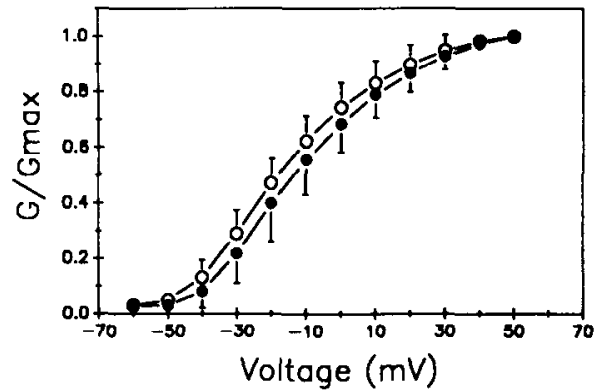

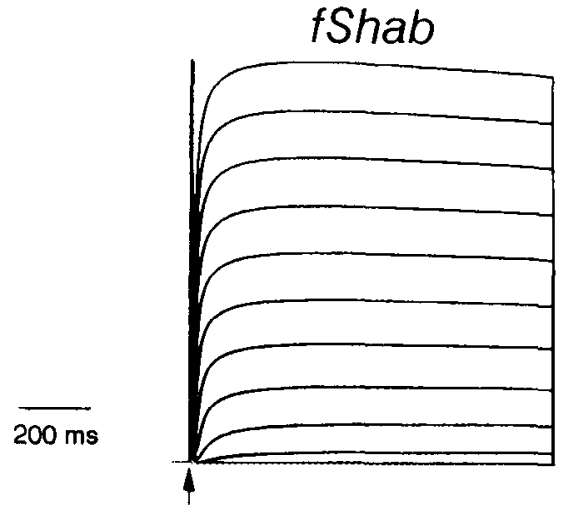
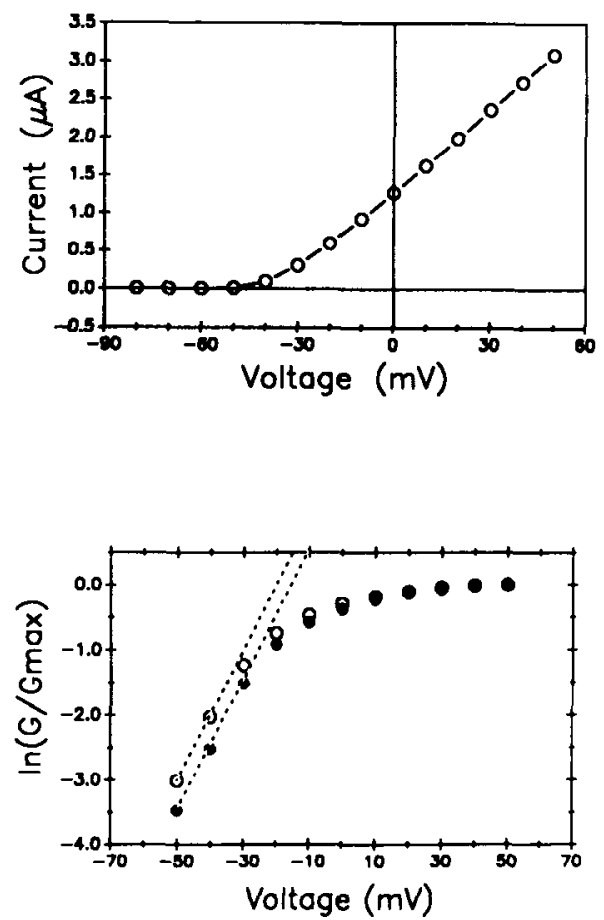

Figure 3. Activation properties of $m S h a b$ and $f S h a b$ currents. A, Comparison of current-voltage relationships of $m S h a b l$ (left) and $f S h a b$ (right) expressed in Xenopus oocytes. Voltage-clamp currents (upper traces) are shown in response to 1 sec depolarizing pulses from a holding potential of $-90 \mathrm{mV}$. The membrane was depolarized in increments of $10 \mathrm{mV}$ until a membrane potential of $+50 \mathrm{mV}$ was reached. Arrows indicate onset of the voltage jump. The currents were linear leak subtracted, but the capacitative current was not subtracted. The interval between trials was 10 sec. Because the $m S h a b l$ current recovers from inactivation more slowly than the $\$ S h a b$ current (see Results), the membrane was hyperpolarized to $-120 \mathrm{mV}$ for $5 \mathrm{sec}$ during the initial half of the interval between trials in $m S h a b 1$ experiments to speed up recovery from inactivation. Because the recovery process is steeply voltage dependent (see Results), virtually all mShabl channels are available for activation at the end of the 5-sec prepulse at $-120 \mathrm{mV}$. The results for $\mathrm{mShabl}$ were the same when the membrane was at $-90 \mathrm{mV}$ during the interval between trials; however, the interval between trials had to be lengthened to allow all the channels to recover from inactivation (15-20 sec). Peak current-voltage relationships are plotted below the current traces. Notice the sharp voltage responsiveness of both channels at approximately $-50 \mathrm{mV}$. $B$, Peak conductancevoltage relationships for $m S h a b l$ (solid circles) and $f S h a b$ (open circles). Left, the peak membrane conductance $(G)$ was calculated for a given command voltage $\left(V_{c}\right)$ and peak current response $\left(I_{\text {peak }}\right)$ from the expression $G=I_{\text {peak }} /\left(V_{c}-V\right)$, where $V$, is the 0 current membrane potential for a given channel. $V$, was measured from tail current analysis and was determined to be between -90 and $-80 \mathrm{mV}$ for all the studied cells (see Fig. 7). Instantaneous current-voltage relationships were approximately linear in the studied voltage range, as determined by similar experiments. $G$ was normalized to the peak membrane conductance at $+50 \mathrm{mV}\left(G_{\max }\right)$. Each symbol is the mean \pm SD of 6 and 10 oocytes injected with $\mathrm{mShabl}$ or $f S h a b$ cRNA, respectively. The membrane reached half-maximal conductance at similar voltages for both $m S h a b l$ and $f S h a b(-14$ and -19 $\mathrm{mV}$, respectively). Right, mean values $\left(G / G_{\max }\right)$ were plotted as $\ln \left(G / G_{\max }\right)$ versus command voltage (Hodgkin and Huxley, 1952a). The slopes (dotted lines) between -50 and $-40 \mathrm{mV}$ were calculated to estimate the limiting equivalent voltage sensitivity of the channels; the activation of both currents appears equally voltage sensitive $(10 \mathrm{mV} / e$-fold $)$. 


\section{Kinetics of Activation}

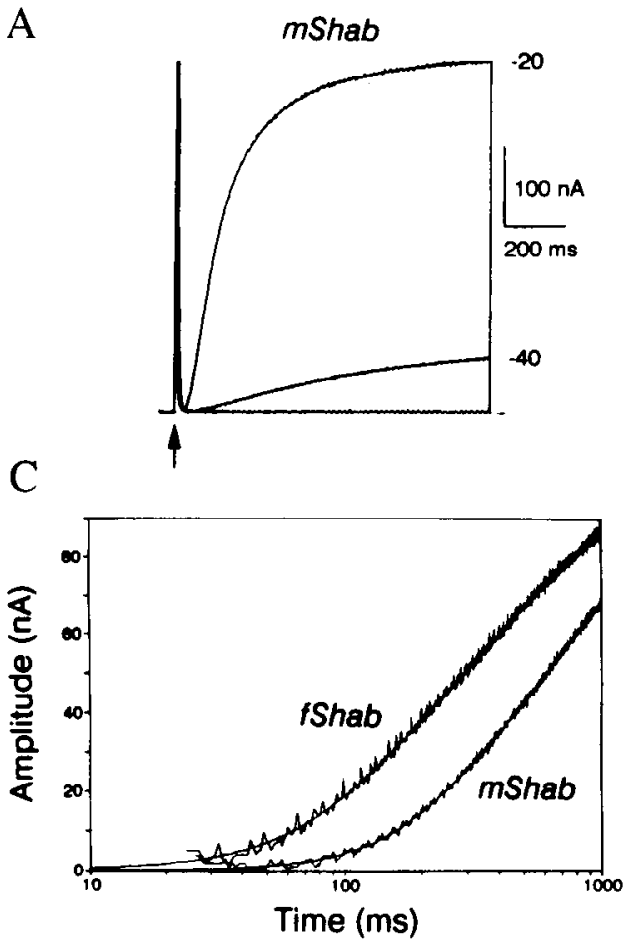

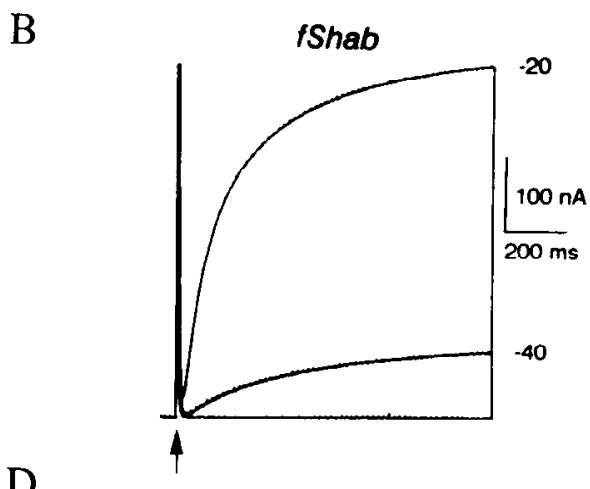

D

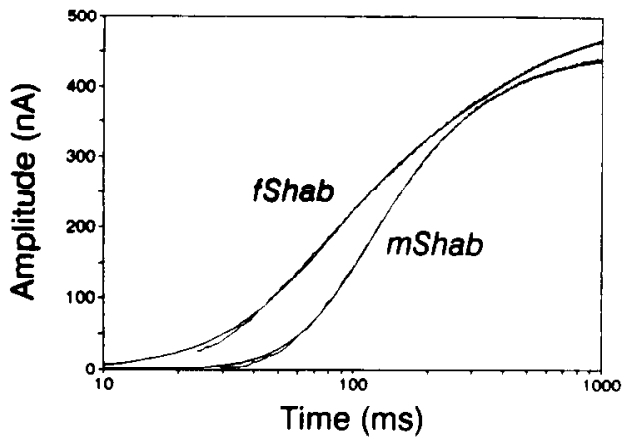

Figure 4. Kinetics of current rise time at low membrane voltages. $A$ and $B$, Current responses to $1-\mathrm{sec}$ depolarizing voltage step pulses from a holding potential of $-90 \mathrm{mV} . A$, mShabl; B, Shab. Arrows indicate pulse onset. Currents were corrected assuming a linear leak; capacitative current was not subtracted. Experiments were carried out at $15^{\circ} \mathrm{C}$. Notice the longer activation delay of $m$ Shabl currents. $C$ and $D$, Current traces from $A$ and $B$ plotted on a $\log$ time scale at $-40 \mathrm{mV}(C)$ and at -20 $\mathrm{mV}(D)$. Lines through the data (excluding the capacitative current) show best fits to a 2-component exponential function,

$I=A_{1}\left[1-\exp \left(-t / T_{1}\right)\right]^{n 1}+A_{2}[1-$ $\left.\exp \left(-t / T_{2}\right)\right]^{n 2}$

where $I$ is the total current response, $A_{1}$ and $A_{2}$ are the amplitudes of the components, and $T_{1}$ and $T_{2}$ are time constants. $m$ Shabl best-fit parameters at $-40 \mathrm{mV}$ are $A_{1}=10.8 \mathrm{nA}, T_{1}=107$ msec, $n 1=5.4, A_{2}=75.2 \mathrm{nA}, T_{2}=$ $519.2 \mathrm{msec}$, and $n 2=1.7$. Shab best fit parameters at $-40 \mathrm{mV}$ are $A_{1}=22.2$ nA, $T_{1}=77.1 \mathrm{mscc}, n 1=2.6, A_{2}=$ $71.7 \mathrm{nA}, T_{2}=405.6 \mathrm{msec}$, and $n 2=$ 1.4. $m$ Shabl best fit parameters at -20 $\mathrm{mV}$ are $A_{1}=279.2 \mathrm{nA}, T_{1}=52.8 \mathrm{msec}$, $n 1=4.9, A_{2}=160.4 \mathrm{nA}, T_{2}=193.4$ $\mathrm{msec}$, and $n 2=2.5$. fShab best fit parameters at $-20 \mathrm{mV}$ are $A_{1}=226.2$ $\mathrm{nA}, T_{1}=45.5 \mathrm{msec}, n \mathrm{l}=2.5, A_{2}=$ $246 \mathrm{nA}, T,=274.8 \mathrm{msec}$, and $n 2=$ 1.3. The higher powers of the exponential terms describing mShabl activation are consistent with a more prolonged delay of current activation (Hodgkin and Huxley, 1952b). between S3 and S4 in both $m S h a b l$ and $f S h a b$ (Fig. 1, $m S h a b$ $283, f$ Shab 530). One consensus site conserved in most $\mathrm{K}^{+}$channel families is found in the segment linking S1 and S2 (Butler et al., 1989); this site, present in $\mathrm{SShab}$, is not found in $\mathrm{mShabl}$ (Fig. 1, fShab 465). It is presently unknown whether native Shab channels are glycosylated.

Potential cAMP-dependent phosphorylation sites (Arg/ $/ \mathbf{L y s}-$ $\mathrm{Arg} / \mathrm{Lys}_{\mathrm{S}}-X-\mathrm{Ser} / \mathrm{Thr}$ or ${ }^{\mathrm{Arg}} / \mathrm{Lys}_{\mathrm{S}}{ }^{\mathrm{Arg}} / \mathrm{Lys}_{\mathrm{s}}-X-X-\mathrm{Ser} / \mathrm{Thr} ;$ Krebs and Beavo, 1979) are present in both $m S h a b l$ and $f S h a b$ on the carboxyl side of the $\mathrm{S} 6$ region. One of these sites is shared by both $\mathrm{mShabl}$ and fShab (Fig. 1; mShab 444, fShab 690), whereas another is at similar but not identical sites ( $m$ Shab 496, Fig. 1; Shab11 731, Butler et al., 1990). The $f$ Shab has a third site (Shab11 796, Butler et al., 1990), whereas the $m S h a b l$ has only 2 potential phosphorylation sites. One or more of these sites are conserved in all $\mathrm{K}^{+}$channels identified to date, which is proposed to be located on the cytoplasmic side in most models (Guy and Conti, 1990).

\section{Functional expression: mShabl and fShab currents have similar current-voltage relations}

To compare the functional properties of $m S h a b l$ and $f S h a b$ currents, Xenopus oocytes were injected with either $m S h a b l$ or $\int S h a b$ cRNA, incubated for approximately $48 \mathrm{hr}$, and then subjected to voltage-clamp analysis using the 2-microelectrode technique. Figure $3 A$ shows $m S h a b l$ and $f S h a b$ currents evoked in response to a family of command pulses from -80 to +50 $\mathrm{mV}$ in $10-\mathrm{mV}$ increments from a holding potential of $-90 \mathrm{mV}$. A comparison of the current-voltage relation of $m S h a b 1$ and $f S h a b$ currents is also shown. For both mShabl and $S$ Shab currents, net (leak subtracted) outward currents were detectable at command voltages more positive than $-50 \mathrm{mV}$, which indicated a similar activation "threshold" for both currents. The current-voltage relation became linear for both $\mathrm{mShabl}$ and fShab currents between -20 and $+40 \mathrm{mV}$.

To obtain additional information about the activation parameters of the currents, the normalized conductance-voltage relations were plottcd for an average of $6(\mathrm{mShabl})$ and 10 $($ Shab) injected oocytes (Fig. 3B, left graph). In both cases, the conductance rose sharply with depolarization and reached its half-maximal value between -15 to $-20 \mathrm{mV}$. By plotting these data on a semilogarithmic graph (Fig. $3 B$, right graph), we found that $m S h a b l$ and $f S h a b$ channels had equal limiting equivalent voltage sensitivities ( $10 \mathrm{mV}$ per $e$-fold change in conductance).

\section{$\mathrm{mShabl}$ current activation is delayed relative to $\mathrm{fShab}$}

Both $m S h a b l$ and $f S h a b$ currents display a relatively slow rate of current activation. However, a closer inspection of the rising phase of the currents at low voltages (Fig. 4) reveals that $m$ Shabl activates more slowly than $f$ Shab. To obtain a relative estimate of the difference, we measured the $10-90 \%$ current rise time at 


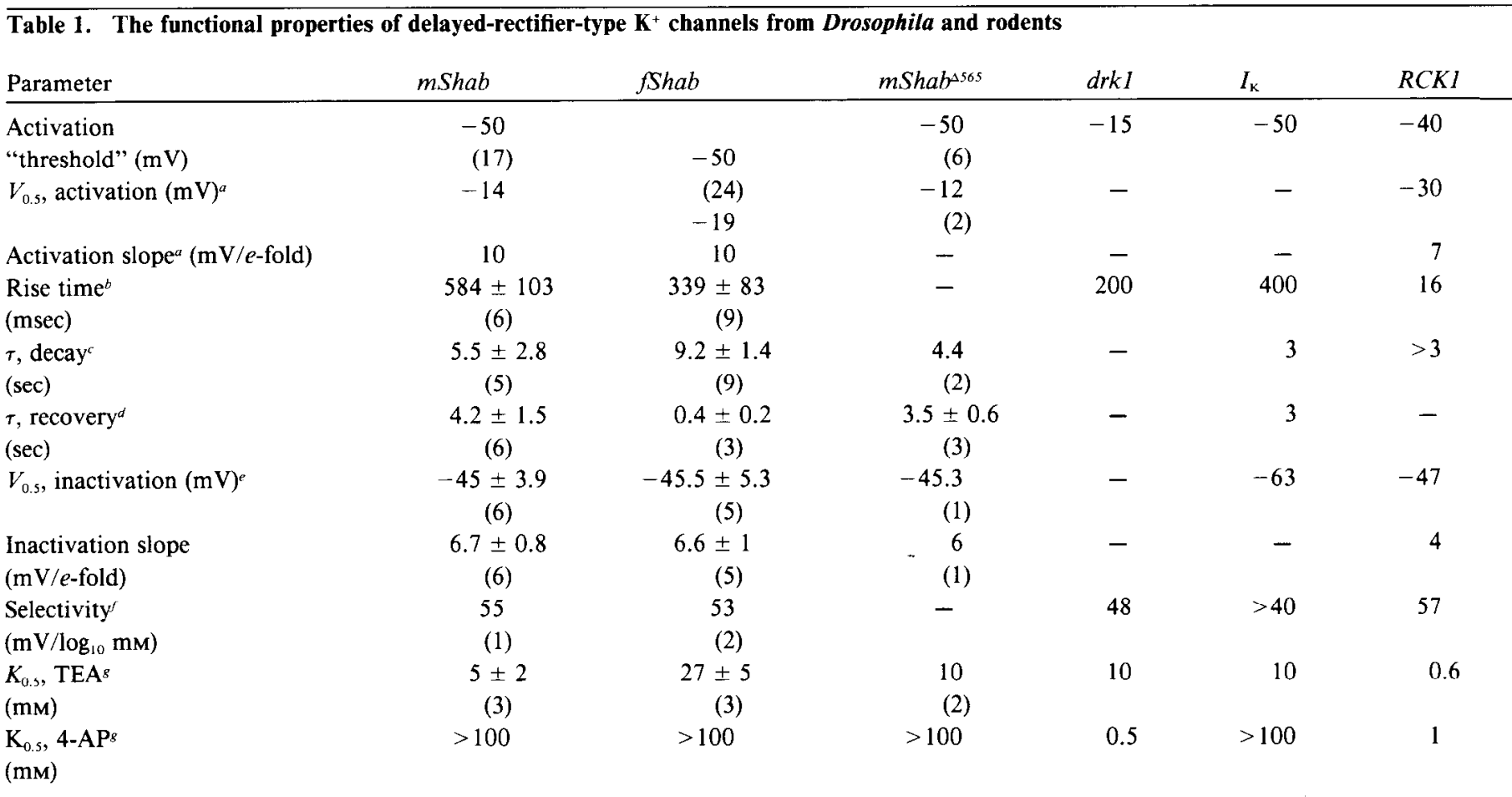

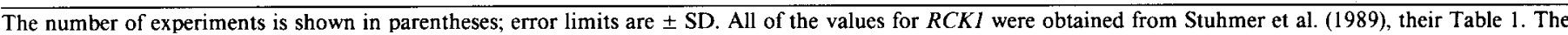
$d r k l$ values are from Frech et al. (1989); $I_{\mathrm{K}}$ values are from Segal and Barker (1984) unless otherwise noted.

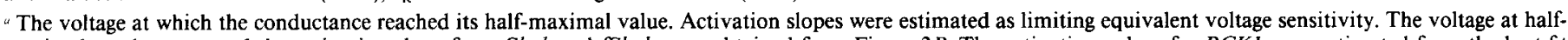

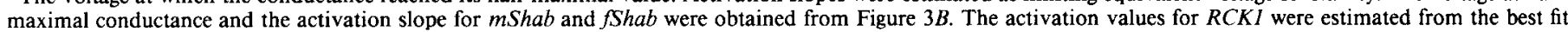
of the conductance-voltage relation to a Boltzmann isotherm (Stuhmer et al., 1989).

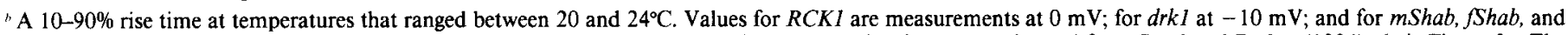

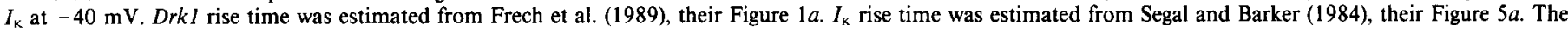
difference between $m S h a b$ and $f S h a b$ was statistically significant at $p<0.001$ (2-tailed Student's $t$ test).

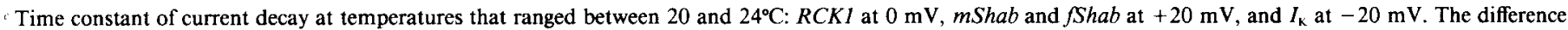
between $m S h a b$ and $f S h a b$ was statistically significant at $p<0.005$ (2-tailed Student's $t$ test).

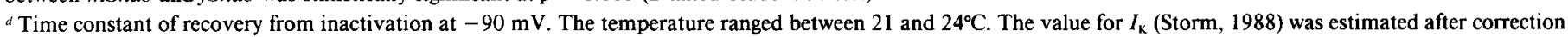
for temperature and voltage dependence (assuming a $Q_{10}$ of 3 and a voltage sensitivity of $28 \mathrm{mV} / e$-fold; see Results).

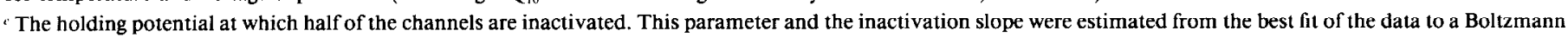
isotherm (see Fig. 5 caption).

Slope of the Nernst theoretical line in $\mathrm{mV}$ per 10 -fold change in external $\mathrm{K}^{+}$concentration.

${ }^{8}$ The concentration of inhibitor necessary to block half of the current (see Fig. 8 ).

$40 \mathrm{mV}$, where slow current activation and the absence of inactivation allow accurate and reproducible measurements. A comparison of the 2 currents showed that $m S h a b l$ required almost twice the time as $f$ Shab to activate (Table 1). This extra time required for $m S h a b l$ current rise is due to slower kinetics of current activation. To describe the time course of current activation (Fig. 4), we fitted the data with an empirical exponential function (Hodgkin and Huxley, 1952b). For both $m$ Shabl and $f S h a b$, the data were best described by the sum of 2 powered exponential terms. However, to describe $m S h a b l$ current activation, the exponential terms had to be raised to a higher power (see Fig. 4 caption). This reflected the substantially longer delay seen in $m S h a b l$ activation (Fig. 4).

For both $m S h a b l$ and $f S h a b$ currents, the powers of the exponential terms needed to describe the delay of current activation were independent of voltage in the range of -40 to -20 $\mathrm{mV}$ (Fig. 4). This was true even though the overall rates of current activation increased at $-20 \mathrm{mV}$ relative to $-40 \mathrm{mV}$. Similar results were obtained at $22^{\circ} \mathrm{C}$; the powers of the exponential terms needed to describe the delay of current activation were the same as in $15^{\circ} \mathrm{C}$ experiments (even though the overall rates of current activation were faster).
Although the kinetics of current rise differ between $m S$ Shabl and $f$ Shab, both currents activate at a rate substantially more slowly than the currents expressed by the mammalian homologs of Shaker, an example of which is $R C K 1$ (Table 1).

mShab1 and Shab currents have similar prepulse inactivation properties, but the rate of recovery from inactivation differs

Both $m S h a b 1$ and $f S h a b$ currents display a clear decay phase during long depolarizing step pulses from a holding potential of $-90 \mathrm{mV}$ (Fig. 6). Although the rates of current decay are slow, they are well described by an exponential function with time constants measured in seconds (Table 1). $m S h a b l$ and $f$ Shab currents showed a consistent difference in this property; when depolarized to $+20 \mathrm{mV}$, the time constant of current decay for $m S h a b 1$ was about half that of $f$ Shab.

Although $m S h a b l$ and $f$ Shab currents are different with regard to macroscopic inactivation rate, the voltage sensitivity of prepulse inactivation is similar. In Figure 5, the best fit to a Boltzmann isotherm indicated that both currents were half-inactivated at about $-45 \mathrm{mV}$; the slope factor of both curves was approximately $7 \mathrm{mV}$ per $e$-fold change in both cases (Table 1).

The rate of recovery from inactivation, however, greatly dif- 


\section{Prepulse Inactivation}

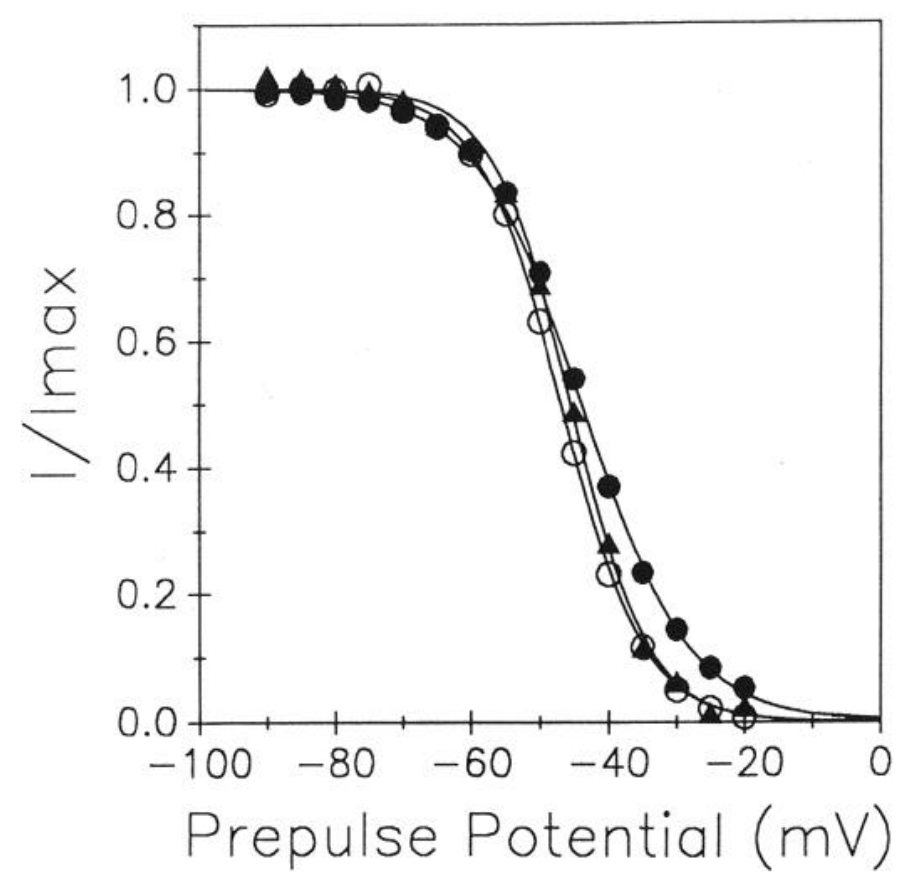

Figure 5. Similar prepulse inactivation properties for mShabl (solid circles), mShabl $1^{\Delta s 65}$ (triangles), and $f$ Shab (open circles). Currents were measured by a test pulse to $+20 \mathrm{mV}$ after a 10 sec prepulse. Prepulses were applied from -90 to $-20 \mathrm{mV}$ in increments of $5 \mathrm{mV}$. In the interpulse interval, the membrane was held at $-90 \mathrm{mV}$ for 10 $\mathrm{sec}$ for $f S h a b$ or for $5 \mathrm{sec}$ at $-120 \mathrm{mV}$ followed by $5 \mathrm{sec}$ at $-90 \mathrm{mV}$ for $m S h a b 1$. The additional interpulse hyperpolarization for $m S h a b l$ was to insure the full recovery of these channels from inactivation (see Results). The solid lines are the best fit to a Boltzmann isotherm:

$$
I=1 /\left(1+\exp \left(\left(V_{h}-V_{t_{h}}\right) / a\right)+b,\right.
$$

where $V_{v_{2}}$ is the prepulse potential $\left(V_{h}\right)$ at which half of the activatable channels are inactivated, $a$ is the slope factor, and $b$ is the amount of current resistant to inactivation. For both $m S h a b l$ and $f S h a b$ channels, $b$ was, on average, about $7 \%$ of the total current [the range was $5-11 \%(n=6)$ and $4-13 \%(n=5)$ for $m S h a b l$ and $f S h a b$, respectively]. After subtracting $b$ from $I$, the difference was normalized to the estimated maximal response $\left(I_{\max }\right)$. Best fit parameters: for $m S h a b l, V_{t}=-46.9 \mathrm{mV}$ and $a=6.3$ $\mathrm{mV} / e$-fold; for $m S h a b 1^{\text {s.56s }}, V_{v_{2}}=-45.3$ $\mathrm{mV}$ and $a=6 \mathrm{mV} / e$-fold; for $f$ Sh $a b, V_{1 /,}$ $=-43.9 \mathrm{mV}$ and $a=7.6 \mathrm{mV} / e$-fold. fers for $m S h a b 1$ and $f S h a b$ currents. This is shown in Figure $6 \mathrm{~A}$, where the response to a second identical voltage pulse is shown after an interval of $1 \mathrm{sec}$. For $f S h a b$, the response to the second pulse is virtually as large as the first. $m S h a b 1$, in contrast, only recovered to about $25 \%$ of its original amplitude in response to the second pulse, which is less than the amplitude of the current at the end of the preceding pulse. This is most likely due to cumulative inactivation (Aldrich et al., 1979), which causes $m S h a b l$ to recover more slowly than $f S h a b$. Indeed, at a holding potential of $-90 \mathrm{mV}$, the recovery from inactivation was almost 10 times faster for $f S h a b$ than for $m S h a b l$ (Fig. $6 B$, Table 1).

Further investigation of recovery from inactivation for $m S h a b 1$ currents showed that the process was both steeply voltage and temperature dependent. Thus, at a more negative holding potential, $-120 \mathrm{mV}$, the time constant decreased 3-fold to $1.4 \pm$
Figure 6. A, Kinetics of current decay and recovery from inactivation. Current traces are responses to twin $10-\mathrm{sec}$ depolarizing pulses separated by an interpulse interval of $1 \mathrm{sec}$. Pulses were to $+20 \mathrm{mV}$ from a holding potential of $-90 \mathrm{mV}$. The 3 currents, mShabl, $m S h a b 1^{\Delta 565}$, and $f S h a b$, all show a slow decay. For $m S h a b 1$ and $m S h a b 1^{\Delta s 65}$, only $20-25 \%$ of the current amplitude recovered during the 1 -sec interpulse interval; $f S h a b$ fully recovered over the same period. $B$, Fraction of total current recovered as a function of interpulse interval duration ( $m$ Shabl, solid circles; mShab1 ${ }^{\Delta 565}$, triangles; fShab, open circles). The holding potential was $-90 \mathrm{mV}$. Continuous lines are best fits to a single exponential function. Estimated time constants are $3.2,2.8$, and 0.3 sec for mShabl, mShabl ${ }^{\Delta s 6 s}$, and $f$ Shab, respectively.
A
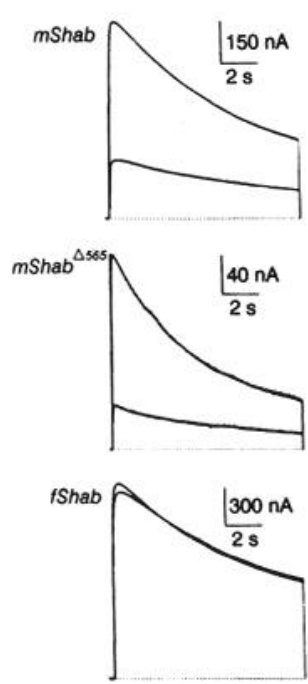

B

\section{Recovery from Inactivation}

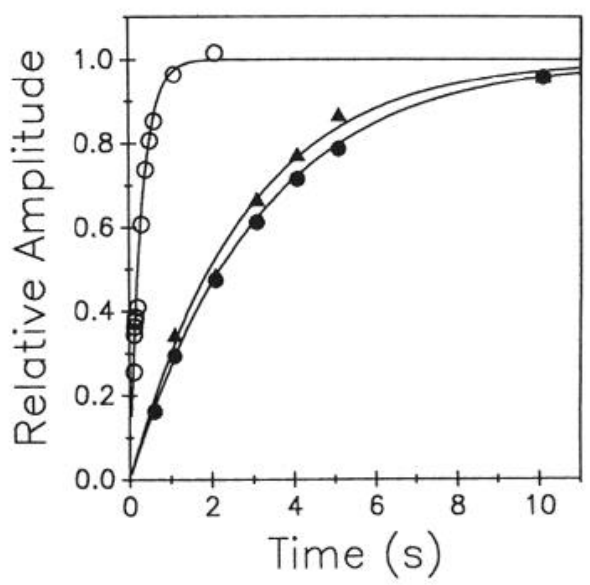




\section{Reversal Potential of Tail Currents}
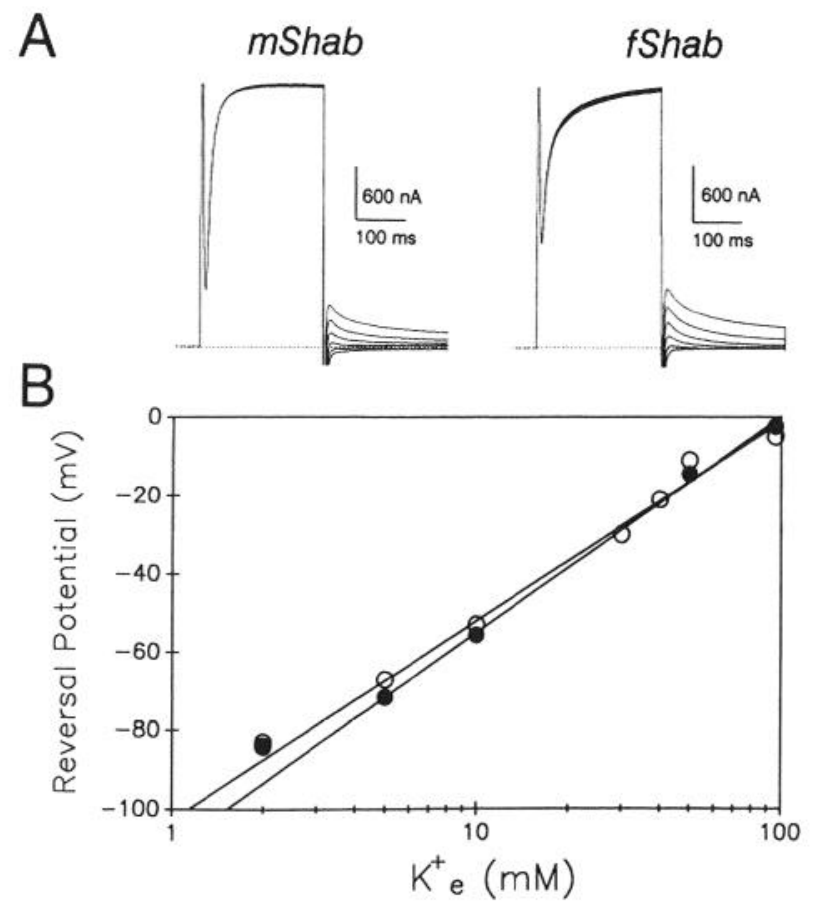

Figure 7. Effect of external potassium ion concentration on current tail reversal potential. $A$, Currents, measured in normal ND96, were elicited by a depolarization to $+50 \mathrm{mV}$ followed by repolarizing steps to potentials from -50 to $-100 \mathrm{mV}$. The dotted line shows the 0 current level. $B$, Tail current reversal potentials are plotted as a function of external $\mathrm{K}^{+}$concentration. The solid lines are the best linear regression fits to the data between 5 and $96 \mathrm{~mm}$ external $\mathrm{K}^{+}$. The slopes are 55 and $51 \mathrm{mV}$ per 10 -fold change in extracellular $\mathrm{K}^{+}$for $m$ Shabl (solid circles) and SShab, (open circles), respectively; both approximate the theoretical Nernst relationship as expected for a $\mathrm{K}^{+}$-selective channel. When external $\mathrm{K}^{+}$concentration was changed, the concentration of $\mathrm{Na}^{+}$ was correspondingly changed, in order to keep extracellular monovalent cation concentration constant.

$0.4 \sec (n=3)$ from $4.2 \pm 1.5 \sec (n=6)$ at $-90 \mathrm{mV}$. At an elevated temperature, $35^{\circ} \mathrm{C}$, the rate of recovery (at $-90 \mathrm{mV}$ ) was approximately $1 \mathrm{sec}$, indicating a relatively high $\mathrm{Q}_{10}$ for this process. A similar temperature dependence was observed for fShab.

\section{Similar ion selectivity and pharmacological properties}

To assess the ion selectivity of the mShabl and $f S h a b$ channels, the reversal potentials of tail currents were measured in different concentrations of external $\mathrm{K}^{+}$. Figure $7 A$ shows an example of current tail reversal in $2 \mathrm{~mm}$ external $\mathrm{K}^{+}$concentration. Figure $7 B$ shows that, as expected for $\mathrm{K}^{+}$-selective channels, the reversal potential became more depolarized in higher external $\mathrm{K}^{+}$. Regression analysis for experiments between 5 and $96 \mathrm{~mm}$ external $\mathrm{K}^{+}$showed slopes of 55 and $53 \mathrm{mV}$ (per 10-fold change in $\mathrm{K}^{+}$concentration) for $m S h a b 1$ and $f S h a b$, respectively (Table 1). These values are close to $58 \mathrm{mV}$, the theoretically expected value for a purely $\mathrm{K}^{+}$-selective channel.

$m S h a b 1$ and $f$ Shab currents were insensitive to 4-AP. Less than $5 \%$ of either current was blocked by $3 \mathrm{~mm} 4$-AP. However, $m S h a b 1$ and $f S h a b$ were both sensitive to tetraethylammonium chloride (TEA), though $\mathrm{mShabl}$ channels were about 5-6 times more sensitive to TEA than $f$ Shab channels (Fig. 8, Table 1).
TEA Sensitivity

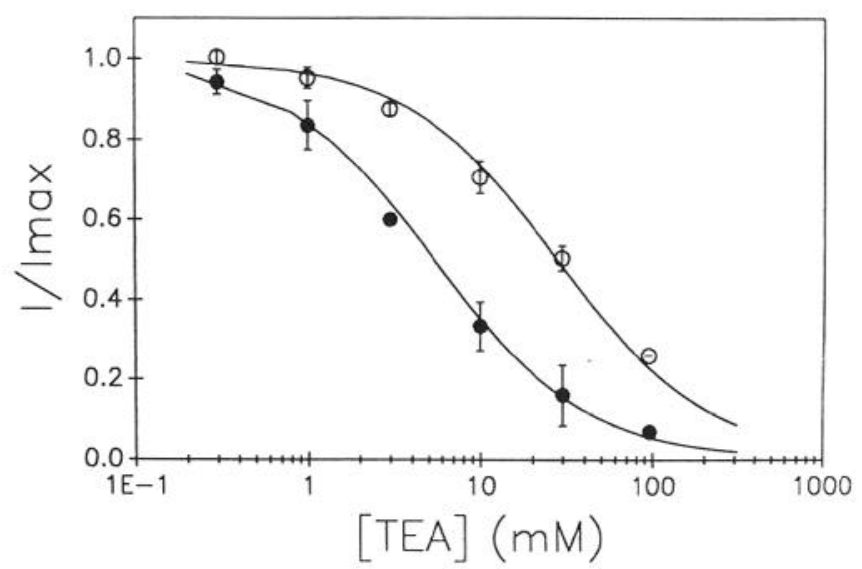

Figure 8. TEA sensitivity of $m S h a b l$ and $f S h a b$ currents. Current amplitude was measured in the presence of variable concentrations of TEA. Data was normalized to a control response in the absence of TEA and plotted versus TEA concentration. Voltage test pulses were to +20 $\mathrm{mV}$ from a holding potential of $-90 \mathrm{mV}$. Each point is the mean $\pm \mathrm{SD}$ of 3 independent determinations. Solid lines are best fits to inhibition isotherms. Estimated TEA concentrations causing a $50 \%$ block were 5 and $27 \mathrm{~mm}$ for $m S h a b 1$ and $f S h a b$, respectively.

\section{The conserved functional properties are determined by the conserved structure}

Although the mShabl and $f$ Shab amino acid sequences have large nonconserved regions outside of the conserved core, the conserved functional properties appear to be determined by structures within or closely flanking the conserved core of the protein. Hence, we created a truncated version of $m S h a b l$ $\left(m S h a b 1^{\Delta 565}\right)$ that consists mainly of the conserved portions of the channel, and observed the functional properties of the current expressed by the shortened form. The mShabl cDNA was cut at the Sph1 site at nucleotide 1691, and a termination codon was added (Figs. 1, 2). This construction removed 293 amino acid residues from the carboxyl end of the protein; most of the remaining 564 amino acids are conserved. Note that the site of truncation in $m S h a b 1$ (Fig. 2, arrow) leaves the region having the conserved hydrophobicity profile intact. The amino terminal region of the shortened clone begins at the normal mShabl initiator methionine, which is only 10 residues upstream from the conserved region (Fig. 1). The shortened clone ends approximately 80 residues downstream from the end of conservation. Analysis of currents expressed by the shortened form of the channel showed that the measured kinetic, voltage-sensitive, and pharmacological properties did not differ significantly from the values shown in Table 1. An example of the similar behavior of this truncated form is shown in Figures 5 and $6 \mathrm{~A}$, which shows similar prepulse inactivation properties, inactivation rate, and rate of recovery from inactivation for $m S h a b l$ and $m S h a b 1^{\Delta 565}$. However, the average current response of oocytes injected with comparable amounts of cRNA was about 10 times smaller for $m S h a b 1^{\Delta 565}$ compared to $m S h a b 1$. The average current amplitude evoked at $+20 \mathrm{mV}$ in the same batch of injected eggs was $0.09 \pm 0.08 \mu \mathrm{A}(n=6)$ for $m$ Shabl ${ }^{\text {s56s }}$ compared to $1.2 \pm 0.6 \mu \mathrm{A}(n=7)$ for $m$ Shabl.

Because the major difference between $m S h a b$ and $f S h a b$ is in the rate of recovery from inactivation, we noted with great in- 
terest that the recovery from inactivation rate was unaffected by the truncation. In previous studies of the Shaker channel (Iverson et al., 1988; Timpe et al., 1988a), the region near the carboxyl terminal had been implicated in determining the rate of recovery from inactivation. Apparently, the sequence removed from $m S h a b l$ does not contain a sequence functionally analogous to that present in the carboxyl terminal region of Shaker clones.

\section{Discussion}

\section{Shab delayed rectifier currents are distinct from other cloned channels}

Most of the mammalian Shaker subfamily $\mathrm{K}^{+}$channels have been categorized as delayed rectifiers because of their slow inactivation, while the fly Shaker current is categorized as an A-current because of its rapid inactivation. However, all Shaker subfamily channels, whether from fly or mammals, share the common property of very rapid current activation, and all are sensitive to 4-AP (Christie et al., 1989; Stuhmer et al., 1989). These properties contrast with those of $m S h a b l$ and $f S h a b$, which have slow activation of macroscopic current and are insensitive to 4-AP. Even a mammalian homolog of Drosophila Shaw that has been expressed (Yokoyama et al., 1989) activates at a rate that is considerably faster than either $m S h a b 1$ or $\int S h a b$. The current-voltage relation of this mammalian Shaw homolog also differs from Shab currents in that it is considerably shifted to depolarized voltages.

The properties of $m S h a b l$ closely resemble those of a native delayed-rectifier-type potassium channel $\left(I_{K}\right)$ in molluscan neurons (Adams et al., 1980) and in rodent hippocampal neurons (Segal and Barker, 1984). Table 1 is a comparison of several properties between $I_{\mathrm{K}}$ from hippocampal neurons and several cloned $\mathrm{K}^{+}$channels. With regard to pharmacological properties, activation "threshold," macroscopic current rise time, current decay, and recovery from inactivation, $m S h a b l$ is the best match. In addition, as seen for $m$ Shabl (Fig. 6A), $I_{\mathrm{K}}$ in vivo also undergoes cumulative inactivation (Aldrich et al, 1979). $I_{\mathrm{K}}$ and $m S h a b$, however, do not seem to match in all measured properties; the major difference is the midpoint of the prepulse inactivation curve (Table 1).

The functional similarities between $I_{\mathrm{K}}$ and $m S h a b l$ are remarkable, but the question may arise as to whether it is valid to compare currents seen in vivo with currents expressed in Xenopus oocytes. Zagotta et al. (1989) showed that Shaker channels behave similarly in vivo and in oocytes. Thus, it is possible that $I_{\mathrm{K}}$ is encoded by the $m S h a b l$ gene, but this remains to be seen.

The high degree of interspecies conservation could be an indication that the functional role of this particular potassium channel is highly defined and restricted to a common role in vertebrates and invertebrates. The main function of $I_{\mathrm{K}}$ could be to provide a delayed repolarization of a slow action potential or to provide a delayed termination of a burst of action potentials (Adams et al., 1980; Hille, 1984). In contrast, the Shaker homologs, which activate more rapidly, could be involved in more rapid phenomena.

\section{Conservation of $\mathrm{mShabl}$ and $\mathrm{fShab}$}

$m S h a b l$ and $f S h a b$ proteins are, in large part, both structurally and functionally conserved, though neither the conserved structural portions nor the functional properties are identical. The conserved portion contains the proposed transmembrane structures of the channels and, in addition, extends into a region upstream towards the amino terminal that is proposed to be cytoplasmic (Tempel et al., 1987). This region is conserved in all members of the $\mathrm{K}^{+}$channel extended gene family (Wei et al., 1990).

The entire extent of conservation between $m S h a b l$ and $f S h a b$ is approximately 470 residues long. Because truncation of the large carboxyl extension of $m S h a b 1$ produced no observable alteration of $m S h a b l$ functional properties, the conserved regions and the regions immediately flanking the conserved region are likely to contain virtually all of the structure that is important for determining the measured biophysical properties of the channel. Indeed, this conserved region is only slightly larger than the area of homology common to all proteins coded by the extended gene family of potassium channels (Wei et al., 1990), and, thus, the similar properties of all voltage-gated potassium channels are likely to be determined by structures within this region. The amino acid substitutions between $m S h a b 1$ and $f S h a b$ that are present in the conserved core of the proteins appear to be relatively neutral with regard to the voltage-sensitive properties common to $m S h a b l$ and $f S h a b$.

Relative to the other potassium channel subfamily members, both $m S h a b l$ and $f S h a b$ are large proteins; $m$ Shabl has an unusually long nonconserved region at the carboxyl end of the protein, while $f S h a b$ is extended on the amino terminal side. The functions of these large regions remain to be determined, but because they do not seem to be involved in determining the kinetic and voltage-sensitive properties that we have measured, other candidate functions may involve subcellular localization or anchoring, pathway of biosynthesis, protein lifetime or turnover rate, or sites of channel modulation. However, the protein sequences we refer to are deduced from the cDNA nucleotide sequence and are based on the assumption that the first AUG of each sequence is the translational start site. The "first AUG rule" holds for hundreds of mRNA sequences that have been analyzed; well over $90 \%$ have translational initiation at the first AUG (Kozak, 1987, 1989). AShabll has polyglutamine immediately downstream from the putative initiator methionine, which is also found in some Shaker proteins (Tempel et al., 1987; Butler et al., 1989). Antibodies directed against a synthetic peptide based on the sequence of this region recognize the Shaker channel protein (Schwarz et al., 1990). Additional evidence that the first AUG is the translational start site comes from an analysis of codon bias; our DNA analysis program (MICROGENIE, Beckman), which analyzes codon usage in all 3 reading frames, suggests a high likelihood that both amino and carboxyl termini are translated regions. Although there is considerable circumstantial evidence that translational initiation is at the first AUG of the largest open reading frame in both species, this has not yet been proven.

In comparing kinetic, voltage-sensitive, and pharmacological properties, perhaps the major difference observed between $m S h a b l$ and $f S h a b$ was the rate of recovery from inactivation. In the Shaker channel, the rate of recovery from inactivation is modified by residues towards the carboxyl end of the protein (Iverson et al., 1988; Timpe et al., 1988a). The difference observed in the rate of recovery between $m S h a b 1$ and $f S h a b$ may also be due to differences at that end of the protein. However, our truncation experiments suggest that, if this is the case, the important structure is likely to be included within the sequence of the shortened version of mShabl. This is because the rate of recovery of the shortened version of $m S h a b l$ was not affected by the truncation.

With regard to the differences in current activation delay be- 
tween $m S h a b l$ and $f S h a b$, it is interesting to note that Hodgkin and Huxley (1952b) suggested that the delay in current activation might be directly related to the number of independent gating particles of a channel, and this number might be reflected in the power of the exponential term needed to describe the delay. However, they also recognized that this might be an oversimplification, and that there might be no such simple relationship between kinetics and structure. Even though the 2 channels, $m S h a b l$ and $f S h a b$, have different activation delays, we assume that these conserved channels have equal numbers of subunits and gate through a mechanistically similar process. Thus, because the exponential terms describing $m$ Sh $a b$ and Sh $a b$ current activation require different powers, the numerical values of these powers are not likely to have a simple relationship to channel structure or gating mechanism.

\section{Mouse and rat Shab $K^{+}$channels: a puzzle.}

One unresolved question centers on the differences observed between $m S h a b l$ and a similar Shah homolog isolated from rat brain. Even though $m S h a b l$ is similar in sequence to $d r k l$, a Shab homolog isolated from rat brain (Frech et al., 1989), $m S h a b l$ is much closer to $S$ Shab in its functional properties than to $d r k 1$. When expressed in Xenopus oocytes, $d r k l$ codes for a $\mathrm{K}^{+}$current that activates at a rate comparable to $m S h a b 1$. However, in contrast to $m S h a b l$ and $f S h a b, d r k l$ channels activate in a voltage range that is more than $30 \mathrm{mV}$ more depolarized. Also distinct are the pharmacological properties of $d r k 1 ; m S h a b l$ and $f$ Shab channels are more than 100 times less sensitive to 4-AP than are $d r k l$ channels (Table 1). The inactivation properties of $d r k 1$ have not been reported, and, thus, a comparison cannot be made.

Two regions differ between $m S h a b l$ and $d r k 1$. Seventeen amino acid differences are present on the carboxyl side of the protein, most of which are downstream from the area of conservation between $m$ Shahl and SShah. A second difference is the putative initiator methionine for $m S h a b 1$, which is 4 amino acids upstream from the initiator methionine of $d r k 1$. Otherwise, the deduced peptides are identical.

Of the 17 amino acid differences on the carboxyl side of $m S h a b 1,14$ are absent in the truncated form of mShab1. Because the truncation of $m S h a b l$ produces no changes in those properties that differ between $m S h a b l$ and $d r k l$, it is unlikely that those 14 residues play a role in distinguishing the functional properties of $m S h a b l$ from $d r k l$. It is possible that the long carboxyl extension, when present in the $d r k l$ form, may interact with the conserved structure of the channel, but does not with the substitutions present in $m S h a b l$. Mutagenesis studies may be necessary to reconcile the functional differences between these 2 channels.

Note added in proof. In light of the recent findings of Mackinnon and Yellen (1990), the fivefold differences in IEA sensitivity between $m S h a b$ and $f S h a b$ could be due to the different residues at miShab 384, Fig. 1.

\section{References}

Adams DJ, Smith SJ, Thompson SH (1980) Ionic currents in molluscan soma. Annu Rev Neurosci 3:141-167.

Aldrich RW, Getting PA, Thompson SH (1979) Inactivation of delayed outward current in molluscan neurone somata. J Physiol (Lond) 291:507-530.

Baumann A, Grupe A, Ackermann A, Pongs O (1988) Structure of the voltage-dependent potassium channel is highly conserved from Drosophila to vertebrate central nervous systems. EMBO J 7:24572463.
Butler A, Wei A, Baker K, Salkoff L (1989) A family of putative potassium channel genes in Drosophila. Science 243:943-947.

Butler A, Wei A, Baker K, Salkoff L (1990) Shal, Shab, and Shaw: three genes encoding potassium channels in Drosophila. Nucleic Acids Res 18:2173-2174.

Chandy KG, Williams CB, Spencer RH, Aguilar BA, Ghanshani S, Tempel BL, Gutman GA (1990) A family of three potassium channel genes with intronless coding regions. Science 247:973-975.

Christie MJ, Adelman JP, Douglass J, North RA (1989) Expression of a cloned rat brain potassium channel in Xenopus oocytes. Science 244:221-224.

Christie MJ, North RA, Osborne PB, Douglass J, Adelman JP (1990) Heteropolymeric potassium channels expressed in Xenopus oocytes from cloned subunits. Neuron 2:405-411.

Connor JA, Stevens CF (1971) Voltage clamp studies of a transient outward membrane current in gastropod neural somata. J Physiol (Lond) 213:21-30.

Frech GC, VanDongen AMJ, Schuster G, Brown AM, Joho RH (1989) A novel potassium channel with delayed rectifier properties isolated from rat brain by expression cloning. Nature 340:642-645.

Grupe A, Schroter KH, Ruppersberg JP, Stocker M, Drewes T, Beckh S, Pongs O (1990) Cloning and expression of a human voltage-gated potassium channel. A novel member of the RCK potassium channel family. EMBO J 9:1749-1756.

Guy RH, Conti F (1990) Pursuing the structure and function of voltage-gated channels. Trends Neurosci 13:201-206.

Hille B (1984) Ionic channels of excitable membranes. Sunderland, MA: Sinauer.

Hodgkin AL, Huxley AF (1952a) Currents carried by both sodium and potassium ions through the membrane of the giant squid axon of Loligo. J Physiol (Lond) 116:449-472.

Hodgkin AL, Huxley AF (1952b) A quantitative description of membrane current and its application to conduction and excitation in nerve. J Physiol (Lond) 117:500-544.

Iverson LE, Tanouye MA, Lester HA, Davidson N, Rudy B (1988) A-type potassium channels expressed from Shaker locus cDNA. Proc Natl Acad Sci USA 85:5723-5727.

Kamb A, Tseng-Crank J, Tanouye MA (1988) Multiple products of the Drosophila Shaker gene may contribute to potassium channel diversity. Neuron 1:421-430.

Koren G, Liman ER, Logothetis DE, Nadal-Ginard B, Hess P (1990) Gating mechanism of a cloned potassium channel expressed in frog oocytes and mammalian cells. Neuron 2:39-51.

Kornfeld R, Kornfeld S (1985) Assembly of asparagine-linked oligosaccharides. Annu Rev Biochem 54:631-664.

Kozak M (1986) Point mutations define a sequence flanking the AUG initiator codon that modulates translation by eukaryotic ribosomes. Cell 44:283-292.

Kozak M (1987) An analysis of 5'-noncoding sequences from 699 vertebrate messenger RNAs. Nucleic Acids Res 15:8125-8148.

Kozak M (1989) The scanning model for translation: an update. J Cell Biol 108:229-241.

Krebs EB, Beavo JA (1979) Phosphorylation-dephosphorylation of enzymes. Annu Rev Biochem 48:923-959.

Kyte J, Doolittle RF (1982) A simple method for displaying the hydropathic character of a protein. J Mol Biol 157:105-132.

Maniatis T, Fritsch EF, Sambrook J (1982) Molecular cloning: a laboratory manual. Cold Spring Harbor, NY: Cold Spring Harbor Laboratory.

McKinnon D (1989) Isolation of a cDNA clone coding for a putative second potassium channel indicates the existence of a gene family. $J$ Biol Chem 264:8230-8236.

Mackinnon D, Yellen G (1990) Mutations affecting TEA blockade and ion permeation in voltage-activated $\mathrm{K}^{+}$channels. Science 250 : 276-279.

Pongs O, Kecskemethy N, Muller R, Krah-Jentgens I, Baumann A, Kiltz HH, Canal I, Llamazares S, Ferrus A (1988) Shaker encodes a family of putative potassium channel proteins in the nervous system of Drosophila. EMBO J 7:1087-1096.

Proudfoot NJ, Brownlec GG (1976) 3' Non-coding region sequences in eukaryotic messenger RNA. Nature 263:211-214.

Saiki RK, Scharf S, Faloona F, Mullis KB, Horn GT, Erlich HA, Arnheim $N$ (1985) Enzymatic amplification of $\beta$-globin genomic sequences and restriction site analysis for diagnosis of sickle cell anemia. Science 230:1350-1354.

Salkoff L (1983) Genetic and voltage-clamp analysis of a Drosophila 
potassium channel. Cold Spring Harbor Symp Quant Biol 48:221231.

Sanger F, Nicklen S, Coulson AR (1977) DNA sequencing with chaintermination. Proc Natl Acad Sci USA 74:5463-5467.

Schwarz TL, Tempel BL, Papazian DM, Jan YN, Jan LY (1988) Multiple potassium-channel components are produced by alternative splicing at the Shaker locus in Drosophila. Nature 331:137-142.

Schwarz TL, Papazian DM, Carretto RC, Jan YN, Jan LY (1990) Immunological characterization of $\mathrm{K}^{+}$channel components from the Shaker locus and differential distribution of splicing variants in Drosophila. Neuron 2:119-127.

Segal M, Barker JL (1984) Rat hippocampal neurons in culture: potassium conductances. J Neurophysiol 51:1409-1433.

Solc CK, Zagotta WN, Aldrich RW (1987) Single-channel and genetic analyses reveal two distinct A-type potassium channels in Drosophila. Science 236:1094-1098.

Storm JF (1988) Temporal integration by a slowly inactivating $\mathrm{K}^{+}$ current in hippocampal neurons. Nature 336:379-381.

Stuhmer W, Stocker M, Sakmann B, Seeburg P, Baumann A, Grupe A, Pongs $O$ (1988) Potassium channels expressed from rat brain cDNA have delayed-rectifier properties. FEBS Lett 242:199-206.

Stuhmer W, Ruppersberg JP, Schroter KH, Sakmann B, Stocker M, Giese KP, Perchke A, Baumann A, Pongs O (1989) Molecular basis of functional diversity of voltage-gated potassium channels in mammalian brain. EMBO J 8:3235-3244.
Swanson R, Marshall J, Smith JS, Williams JB, Boyle MB, Follander $\mathrm{K}$, Luneau CJ, Antanavage J, Oliva C, Buhrow SA, Bennett C, Stein RB, Kaczmarek LA (1990) Cloning and expression of cDNA and genomic clones encoding three delayed rectifier potassium channels in rat brain. Neuron 4:929-939.

Tempel BL, Papazian DM, Schwarz TL, Jan YN, Jan LY (1987) Sequence of a probable potassium channel component encoded at Shaker locus of Drosophila. Science 237:770-775.

Tempel RI., Jan YN, Jan I.Y (1988) Cloning of a probable potassium channel gene from mouse brain. Nature 332:837-839.

Timpe LC, Jan YN, Jan LY (1988a) Four cDNA clones from Shaker locus of Drosophila induce kinetically distinct $\Lambda$-type potassium currents in Xenopus oocytes. Neuron 1:659-667.

Timpe LC, Schwarz TL, Tempel BL, Papazian DM, Jan YN, Jan LY (1988b) Expression of functional potassium channels from Shaker cDNA in Xenopus oocytes. Nature 331:143-145.

Wei A, Covarrubias M, Butler A, Baker K, Pak M, Salkoff L (1990) Potassium current diversity is produced by an extended gene family conserved in Drosophila and mouse. Science 233:780-782.

Yokoyama S, Imoto K, Kawamura T, Higashida H, Iwabe N, Miyata T, Numa $S$-(1989) Potassium channels from NG108-15 neuroblastoma-glioma hybrid cells. FEBS Lett 259:37-42.

Zagotta WN, Hoshi T, Aldrich RW (1989) Gating of single Shaker channels in Drosophila muscle and in Xenopus oocytes injected with Shaker mRNA. Proc Natl Acad Sci USA 86:7243-7247. 